\title{
A propos de la prostate
}

\section{Eberhard Wolff}

Prof. Dr rer. soc., rédacteur culture, histoire, société

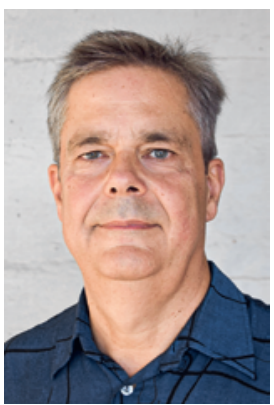

Je m'en souviens très bien: il y a plusieurs dizaines d'années, notre professeur de chimie vieillissant s'est vanté tout à trac de l'état impeccable de sa prostate devant la classe. Fraîchement examinée et de la taille d'une noix. Autant dire que pour des adolescents d'un lycée de garçons comme nous, cette sortie inattendue était des plus embarrassantes.

Un livre, lié en quelque sorte à cette histoire farfelue, vient de paraître [1]. Un recueil en allemand de textes de spécialistes suédois en «Medical Humanities» nous permet de mieux comprendre cette glande. Il montre que la prostate est en fait bien plus qu'une glande à l'origine de potentiels problèmes de santé tels que la prostatite, l'hyperplasie ou les carcinomes.

Peut-être notre professeur de l'époque voulait-il déjà nous convaincre de l'intérêt du principe de précaution. Aujourd'hui, le cancer de la prostate détecté à un stade précoce, comme chez l'acteur hollywoodien Ben Stiller, sert à vanter les mérites et le succès de la prévention. Mais cette dernière n'est plus considérée uniquement comme une bonne chose. Le dépistage détecte également de nombreux malades potentiels, détermine les sujets à risque, suscite des émotions, établit de nouvelles normes comportementales et conduit parfois au surtraitement. Ces dernières années, le BMS s'est souvent fait l'écho des réserves quant à l'usage irréfléchi des tests PSA.

La prostate était pour nous, jeunes élèves, un embarras urogénital et elle le reste aujourd'hui. Son examen peut provoquer «honte, gêne, vulnérabilité», voire des états anxieux, surtout chez les hommes qui ont été victimes d'abus sexuels. Le personnel médical réagit en général par une sorte de précaution routinière. Toutefois les manuels suédois, en tout cas, ne sont pas d'un grand secours, notamment quant à la position d'examen la plus adaptée.

Peut-être notre professeur de chimie voulait-il simplement prouver sa virilité. Aujourd'hui encore, la prostate est étroitement liée à une image conventionnelle de l'homme, dont fait partie le contrôle de son propre corps, donc de ses fonctions urinaires. En plus d'être très pénibles, les problèmes dus à une hypertrophie de la prostate égratignent aussi cette vision de la virilité En Allemagne, l'expression péjorative «vessie de confirmand" désignait autrefois le besoin fréquent d'uriner. Les traitements chirurgicaux peuvent entraîner des troubles de l'érection. Les traitements hormonaux "féminisent» les patients. Après une opération, ceux-ci doivent souvent se trouver une nouvelle identité en tant qu'hommes. Peut-être notre professeur avait-il, comme beaucoup de patients aujourd'hui, peur de perdre sa virilité.

Ou peur de la menace d'une procédure chirurgicale douloureuse. Un autre souvenir me revient brusquement: je crois que c'est notre professeur de religion qui, de manière subtilement provocative, nous a décrit le douloureux «rabotage» d'une prostate hypertrophiée. Peut-être notre professeur de chimie vieillissant voulait-il, par cette déclaration inattendue, nous prouver qu'il restait jeune. Quand on "pisse comme un vieux», on ne remplit en effet plus les normes habituelles. Ces dernières transparaissent même dans le paysage urbain; il suffit de regarder l'espace entre les pissoirs publics. Et de façon générale, les hommes (blancs) âgés n'ont pas la cote aujourd'hui.

Enfin, peut-être notre professeur de chimie voulait-il nous prouver sa normalité morale. Au début du $\mathrm{XX}^{\mathrm{e}}$ siècle, les problèmes de prostate étaient également associés à des pratiques sexuelles «anormales» et «immorales», comme les aventures extraconjugales et la masturbation. Un pan d'histoire médicale pourrait donc aussi avoir joué un rôle pendant ce cours.

Ce recueil passionnant montre tout ce qui dépend de cette "glande unique». Nos enseignants de l'époque ont abordé la question de façon maladroite et inadéquate. Brian Regli, un patient américain atteint d'un cancer, qui a composé une chanson d'adieu à sa prostate avant l'ablation de celle-ci en 2015 et l'a postée sur YouTube [2], s'est mieux débrouillé. A voir absolument!

1 Björkman M (ed.). Der Mann und die Prostata. Kulturelle, medizinische und gesellschaftliche Perspektiven. Bielefeld: transcript; 2019. ISBN 978-3-8376-4866-9. € 19.90. Cet «Et encore» s'appuie largement sur cet ouvrage.

2 Brian Regli: "Goodbye to my prostate».

https://www.youtube.com/watch?v=SWBnR_8yTBo 